\title{
TV/Series
}

$2 \mid 2012$

Les séries télévisées dans le monde : Échanges, déplacements et transpositions

\section{Du Mexique à l'île de La Réunion : Études de réception de deux telenovelas " créolisées »}

\section{Éliane Wolff}

\section{(2) OpenEdition \\ Journals}

Édition électronique

URL : http://journals.openedition.org/tvseries/1427

DOI : 10.4000/tvseries. 1427

ISSN : 2266-0909

Éditeur

GRIC - Groupe de recherche Identités et Cultures

Référence électronique

Éliane Wolff, «Du Mexique à l'île de La Réunion : Études de réception de deux telenovelas

" créolisées » », TV/Series [En ligne], 2 | 2012, mis en ligne le 01 novembre 2012, consulté le 19 avril 2019. URL : http://journals.openedition.org/tvseries/1427 ; DOI : 10.4000/tvseries.1427

\section{(c) (i) $\ominus$}

$T V /$ Series est mis à disposition selon les termes de la licence Creative Commons Attribution - Pas d'Utilisation Commerciale - Pas de Modification 4.0 International. 


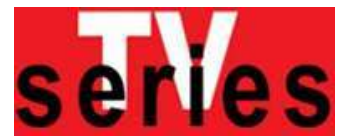

\title{
Du Mexique à l'île de La Réunion : Études de réception de deux telenovelas « créolisées 》

\begin{abstract}
Les responsables du Réseau France Outre-mer ont choisi de promouvoir la diffusion de telenovelas de type "romance ", persuadés que ces programmes produits en Amérique Latine sont "plus adaptés aux publics d'Outre-mer que les fictions françaises et européennes voire américaines ». De fait, ces produits recueillent, depuis de nombreuses années, de très bonnes audiences auprès du public ultramarin. Pour répondre au plus près aux attentes de son public, le réseau a fait une succession de paris, parmi lesquels la traduction des dialogues de Rosalinda en créole réunionnais et la production de La Baie des Flamboyants (une adaptation d'une telenovela mexicaine, Código postal), dans les décors tropicaux de la Guadeloupe, avec des comédiens originaires des DOM. Ce choix des diffuseurs devenus co-producteurs n'a pas reçu l'accueil espéré. Dans cet article nous nous interrogerons dans un premier temps sur les raisons du succès des séries telenovelas à l'île de La Réunion. Puis nous proposerons quelques pistes pour comprendre la réception des adaptations "créolisées » des deux telenovelas mexicaines Rosalinda et Código postal produites par Televisa. Comment saisir les publics et faire entendre leurs voix sans tomber dans le travers de la " ventriloquie » ? Une question que l'on tentera de traiter dans ce texte situé résolument dans une sociologie des médias et de la réception.
\end{abstract}

$\mathrm{P}$ roduites par les industries culturelles des pays d'Amérique latine pour leurs publics populaires nationaux (Brésil et Mexique en particulier), les telenovelas ont longtemps été boudées par le public hexagonal $^{1}$ et stigmatisées en tant que sous-produit de la culture de masse aux contenus formatés et aliénants. Comment définir ce produit audiovisuel ? La telenovela raconte une histoire qui, le plus souvent, finit bien, et s'adresse à des publics cibles divers. Longtemps réduites à la seule " romance ${ }^{2}$ ", les telenovelas traitent, en fait, une pluralité de thèmes qui définissent autant de genres différents : on distingue les telenovelas historiques qui intègrent des éléments d'archives, les adaptations d'œuvres littéraires (comme Escrava Isaura, adaptation du roman de Bernardo Guimarães, voir figure 1), les telenovelas pro-sociales évoquant des questions sociétales comme le contrôle des naissances, l'homosexualité, la maladie et la greffe de moelle osseuse (comme Laços de familia, voir figure 2) ou politiques avec la présence des mafias (comme Hombres de Honor, voir figure 3) et des débats sur le travail clandestin.

\footnotetext{
1 Voir Philippe Bouquillon, "La réception des telenovelas brésiliennes en France ", Intercom, vol. $\mathrm{XV}, \mathrm{n}^{\circ} 1,1992$, p. 98-117.

${ }^{2}$ Telenovela traditionnelle "à l'eau de rose ", dont l'hérö̈ne est souvent une jeune fille pauvre qui, après bien des épreuves, finit par trouver l'amour et le bonheur.
} 


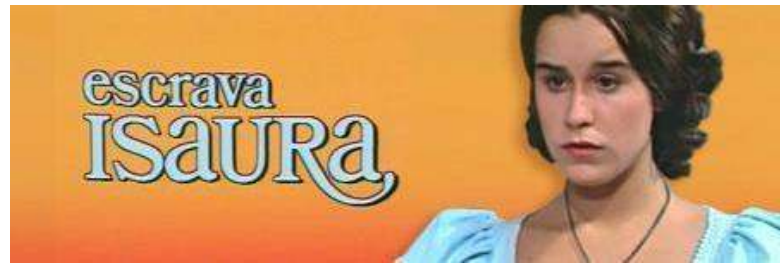

Fig. 1 : Escrava Isaura (Brésil, Globo, 1976)

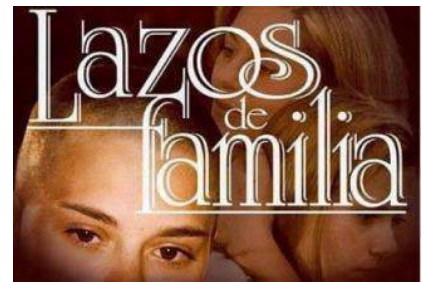

Fig. 2 : Laços de familia (Brésil, Globo, 2000)

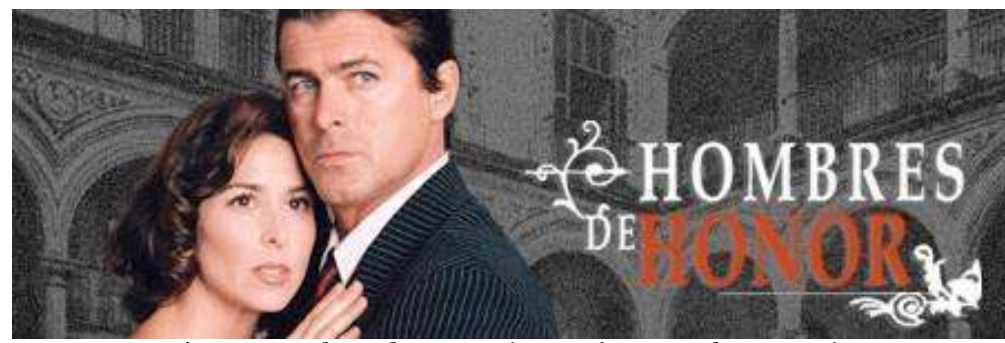

Fig. 3 : Hombres de Honor (Argentine, Canal 13, 2005)

Ce produit audiovisuel relève donc d'un genre complexe, investi de fonctions sociales précises, comme a pu le montrer Erika Thomas ${ }^{3}$ dans le cas de la telenovela brésilienne. Ces séries s'exportent désormais à l'international dans plus d'une centaine de pays et ont conquis progressivement de très nombreux publics ${ }^{4}$, en particulier celui des départements et des territoires français ultramarins, qui enregistrent de très fortes audiences depuis plus d'une vingtaine d'années. Le Réseau France Outre-mer (RFO), positionné comme la

3 Voir Erika Thomas, Les Telenovelas entre fiction et réalité, Paris, L’Harmattan, 2003 ;

"Les telenovelas : une passion brésilienne », Ina Global, La Revue des industries créatives et des médias (lien consulté le 20 juin 2011) :

http://www.inaglobal.fr/television/article/les-telenovelas-une-passion-

bresilienne?tq=12\#intertitre-2

${ }^{4}$ La France n'a découvert le genre que depuis peu avec la diffusion sur M6 en 2005 de Rubi

(Mexique, Televisa, 2004). 
télévision de la diversité et de l'Outre-mer5, a fait de la diffusion des telenovelas une priorité, persuadé que ces séries sont "plus adaptées aux publics d'Outre-mer que les fictions françaises et européennes voire américaines ${ }^{6} »$. Pour répondre encore mieux aux attentes « supposées7 " des publics de ses neuf stations régionales (voir figure 4), le réseau a fait une succession de paris afin de créoliser la telenovela : traduction et relocalisation de la production visent ainsi à lui donner une couleur plus domienne. Ce choix des diffuseurs désormais co-producteurs a pour objectif premier d'augmenter l'audience face à la concurrence des chaînes privées qui se disputent les faveurs des téléspectateurs à coup de programmations de telenovelas.

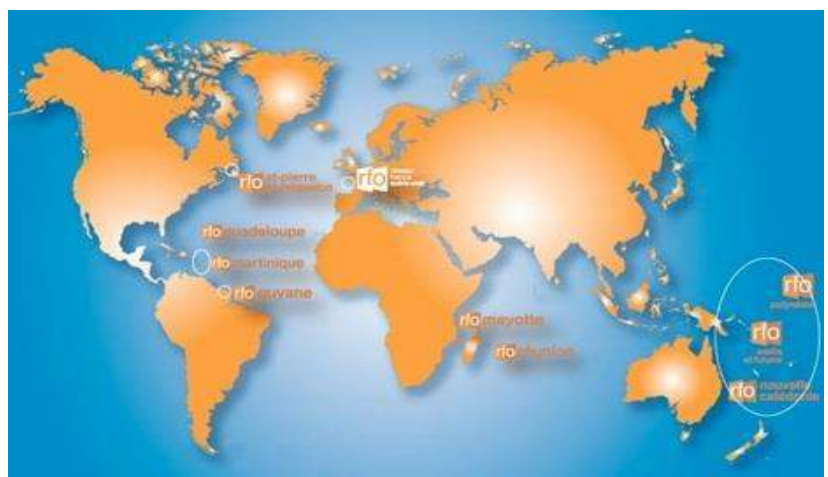

Fig. 4 : RFO et ses stations régionales

Dans cet article nous tenterons de décrypter, dans un premier temps, les raisons du succès des séries telenovelas à l'île de La Réunion; puis nous proposerons quelques pistes de compréhension de la réception des adaptations "créolisées" de deux telenovelas mexicaines, Rosalinda (Televisa, 1999) et Código postal (Televisa, 2006), qui n'ont pas reçu l'accueil espéré. Comment saisir les publics et faire entendre leurs voix sans tomber dans le travers de la " ventriloquie » dénoncée par Dayan ${ }^{8}$ ? Une question que l'on tentera de traiter dans ce texte situé résolument dans une sociologie des médias et de la réception?.

5 Intégré en 2004 au groupe France Télévision, RFO devient Réseau Outre-Mer $1^{\text {re }}$ en 2010. ${ }^{6} \mathrm{CSA}$, Bilan de la société nationale de programme Réseau France outre-mer, 2002, p. 42. 7 Ibid.

${ }^{8}$ Daniel Dayan, « Les mystères de la réception. Ethnologues, missionnaires, ventriloques », Le Débat, $\mathrm{n}^{\circ} 71,1992 / 4$, p. 150 [p. 146-162].

${ }^{9}$ Éliane Wolff, "Publics, réception médiatique et contexte d'usage : retour sur 10 années de recherches » in Bernard Idelson (dir.), Journalismes dans l'Océan Indien: espaces publics en questions, Paris, L'Harmattan, 2008, p. 53-67. 


\section{Les raisons d'un engouement pour les telenovelas}

La vie quotidienne réunionnaise a été scandée pendant l'année 2001 par la diffusion d'une telenovela mexicaine, Marimar $^{10}$, et a vu la mobilisation sans précédent d'un public qui réclamait sa rediffusion à une heure de grande écoute. Parce que Thalia, l'actrice interprétant le personnage principal de Marimar (voir figure 5), avait suscité une très forte identification, il était courant d'entendre les téléspectateurs affirmer lors de nos enquêtes ${ }^{11}$ et des micros-trottoirs effectués par la station régionale : " Marimar, c'est une créole, c'est notre histoire ». Nos premières recherches nous ont permis de mieux comprendre le formidable succès de cette telenovela à La Réunion, auprès de publics auxquels ce produit n'était pas destiné a priori ${ }^{12}$. Les raisons de cet engouement sont à chercher du côté de la proximité historique et socioculturelle entre l'Île de La Réunion et les pays latino-américains, producteurs de ces séries.

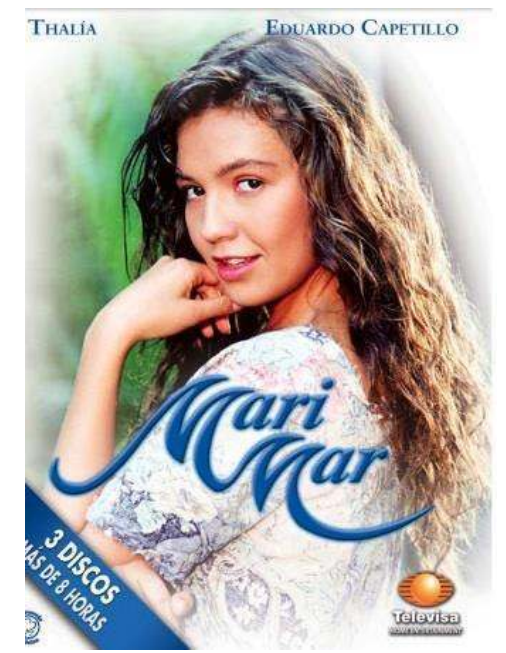

Fig. 5 : Marimar (Televisa, 1994)

\footnotetext{
${ }_{10}$ Produite par le groupe Mexicain Televisa en 1994, Marimar est une telenovela de type romance de 149 épisodes diffusée en 2001 à La Réunion, où elle a obtenu des audiences jamais atteintes jusque-là.

11 Des entretiens ont été menés durant plusieurs années (2002-2009) auprès de téléspectateurs réunionnais dans le cadre, en particulier, d'une formation à la pratique de la recherche auprès d'étudiants de la filière Information-Communication.

${ }^{12}$ Voir Éliane Wolff, « De Marimar à Rosalinda péi (pays), l'expérience réunionnaise », in Eric Maigret et Guillaume Soulez (dir.), Les Raisons d'aimer les séries télé, MédiaMorphoses, numéro spécial, janvier 2007, p. 59-63.
} 
On sait, depuis les études pionnières sur la réception des feuilletons ${ }^{13}$, que les publics "s'interprètent plus qu'ils n'interprètent ${ }^{14}$ », en fonction de leurs origines et de leurs ressources culturelles, mais également en raison de leur proximité vis-à-vis de l'espace de production. Or, même si leur histoire n'est pas la même, La Réunion et l'Amérique latine ont beaucoup de points communs, parmi lesquels la colonisation, l'importation d'Afrique d'une main d'œuvre servile, un système d'organisation sociale spécifique. Certes, la constitution des propriétés foncières s'est faite différemment dans les deux espaces: à La Réunion, une île vierge de toute occupation jusqu'au milieu du XVII ${ }^{\mathrm{e}}$ siècle, les terres ont été concédées au nom du Roi par la Compagnie des Indes, alors qu'en Amérique du Sud, c'est après la spoliation des terres indiennes que les colons se sont trouvés à la tête d'immenses propriétés. Mais dans les deux cas, "habitations " ici ou «haciendas » là-bas comprennent à la fois les terres, les cultures, le logement des maîtres et des travailleurs, et renvoient à un système social spécifique et commun aux deux entités : la plantation. Le métissage est une autre constante partagée de part et d'autre. En Amérique, le manque de femmes blanches a rapidement conduit les colons à s'unir à des femmes indiennes ou africaines. Ce métissage s'est poursuivi malgré la désapprobation dont il fit l'objet ensuite, en particulier pour les unions entre blancs et noirs. À La Réunion, où le peuplement s'est fait par apports successifs en provenance de Madagascar, d'Europe, d'Inde du Sud tamoule et d'Inde du Nord musulmane, de Chine et plus récemment de Mayotte et des Comores, le métissage fait partie de la réalité quotidienne, même s'il reste marqué par des unions préférentielles ${ }^{15}$. Enfin, ces deux entités apparaissent en profonde transformation, passant très rapidement d'une organisation de type agricole à une société hypermoderne, branchée sur les réseaux de communication (c'est particulièrement le cas pour La Réunion) et développant des industries culturelles qui, dans le cas de l'Amérique latine, diffusent leurs produits dans le monde entier. Ces mutations rapides ont vu l'émergence d'une classe moyenne porteuse de nouveaux modèles de comportements, mais dont le mode de vie reste très hybride, marqué à la fois par la tradition et la modernité. De part et d'autre, la religion catholique est dominante, même si elle coexiste avec d'autres pratiques magico-religieuses, et le développement du nombre de laissés-pour-compte du progrès est important.

13 Voir Tamar Liebes et Elihu Katz, The Export of Meaning: Cross-Cultural Readings of 'Dallas', New York, Oxford University Press, 1990.

14 Jean Bianchi, «La promesse du feuilleton. Structure d'une réception télévisuelle », Réseaux, $\mathrm{n}^{\circ} 39,1990$, p. 9-18

15 Éliane Wolff, « Familles en mutations, famille en questions » in Michel Watin (dir.), La Réunion, une société en mutation, Univers créoles 7, Paris, Economica-Anthropos, 2010, p. 81-102. 
Ces profondes similitudes entre des entités si éloignées géographiquement peuvent expliquer en partie l'engouement important des Réunionnais pour les telenovelas, en particulier les romances qui les font rêver ou leur parlent d'eux et de leur vie :

Marimar était comme nous dans le temps lontan [...]. Elle portait de vieux linges rapiécés, elle marchait pieds nus et elle vivait dans une vieille petite case, sans eau ni électricité, avec son grand-père et sa grand-mère. Elle lavait son linge à la rivière avec un savon et des galets ... Nous aussi nous avons connu cette vie de misère ${ }^{16}$.

Ces séries mettent en scène des personnages métissés, des décors et paysages familiers, une nature tropicale connue, une bande son et des chants appréciés, et traitent de thèmes en résonance profonde avec le vécu et les attentes des téléspectateurs réunionnais. Les rapports entre les riches et les pauvres, ainsi que le mariage qui permet de sortir de sa condition, connaissent un écho tout particulier ici, notamment lorsque l'union transgresse les barrières raciales, religieuses, ethniques ou sociales. Les questions identitaires et l'évocation des liens familiaux marqués par la vengeance, la jalousie et la rumeur (le ladi lafé en créole réunionnais) sont très appréciées et provoquent débats et commentaires. L'émotion est grande lorsque les images évoquent un passé marqué par l'esclavage, la misère, la faim, l'oppression des petits propriétaires, le racisme, les humiliations, la dureté de la vie sur l'exploitation. "Les gens nous racontaient leur vie passée en la comparant à celle de Marimar. Ils pleuraient quand ils nous racontaient cette histoire, c'était leur histoire ", témoigne une assistante de production ${ }^{17}$, jusque-là plutôt méprisante du genre comme nombre de ses collègues à la station régionale. Ce regard négatif a été transformé par la rencontre avec le public réel au cours de reportages au domicile de téléspectateurs et de micros-trottoirs visant à rendre compte de l'engouement persistant pour une telenovela devenue véritable phénomène de société dans l'île. Forte de ce succès d'audience, RFO, jusque-là diffuseur, a parié sur le genre en diffusant beaucoup de séries de ce type et a multiplié les initiatives afin de répondre au plus près aux attentes de «ce public imaginé ${ }^{18}$ » en créolisant les telenovelas. Ces initiatives n'ont pas forcément été bien accueillies et ont suscité controverses, débats et conflits de définitions dans la sphère publique.

\footnotetext{
16 Entretien paru dans le Quotidien de La Réunion, 2 novembre 2010, dossier " Telenovelas. Pourquoi La Réunion en est dingue », p. 8.

17 Entretien recueilli dans le cadre du mémoire de maîtrise en SIC de Stéphanie Hoareau, " À la recherche des publics de Marimar à la Réunion », dirigé par Éliane Wolff, Université de la Réunion, 2005.

${ }^{18}$ Eric Macé, «Qu'est-ce qu'une sociologie de la télévision ? (2) Esquisse d'une théorie des rapports sociaux médiatisés. Les trois moments de la configuration médiatique de la réalité : production, usages, représentations. ", Réseaux, n¹05, 2001, p. 199-242.
} 


\section{Les paris de la créolisation des telenovelas}

\section{a) La traduction en créole}

L'initiative du premier pari appartient à la station régionale de La Réunion qui a proposé la traduction en créole des épisodes de la telenovela mexicaine Rosalinda, interprétée par Thalia, l'actrice fétiche des Réunionnais (voir figure 6) :

Puisque les gens ont aimé le feuilleton, on s'est dit que de le proposer en créole c'était une bonne idée ... mettre le créole à l'antenne et faire adhérer le public en proposant du créole sur un feuilleton qu'ils aiment. Donc on avait deux atouts : une série qu'ils aiment, et en créole qui est la langue que tout le monde parle [...]. C'était un pari, c'est vrai qu'on n'est jamais sûr de rien ${ }^{19}$.

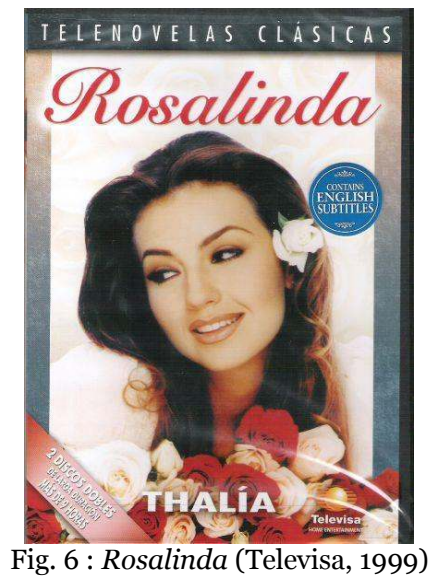

L'initiative a suscité un rejet massif : les audiences ont chuté brutalement à la grande surprise des promoteurs et l'expérience a été très rapidement arrêtée. Pourquoi un tel rejet ? Les hypothèses sont multiples. Sont évoqués le format, passé de 26 à 13 minutes, et la rediffusion, qui émousse l'intérêt pour une histoire dont on connaît le dénouement. Par ailleurs, la traduction a été réalisée avec de faibles moyens, dans un temps relativement court et avec un amateurisme certain : il n'y a pas eu de véritable casting pour choisir les voix, puisque la distribution s'est faite dans le cadre d'un entre soi familial ${ }^{20}$. De plus, ces non-professionnels doublaient plusieurs personnages et les voix étaient mal synchronisées, en décalage avec le mouvement des lèvres. Cependant de telles imperfections sont également courantes

19 Entretien avec G. Prufer, directeur de la station RFO Réunion (novembre 2006)

${ }^{20}$ Le directeur du studio a ainsi mobilisé famille et amis. 
dans le cas des doublages en français ${ }^{21}$ et n'ont jamais été un frein au succès des telenovelas. Il faut chercher ailleurs les causes de ce rejet brutal, en particulier du côté de la traduction en créole. On note le manque total de variations dans les registres de langues convoqués. La traduction ne tient pas compte des situations d'interactions, du contexte d'énonciation et encore moins du statut des locuteurs. La jeune Rosalinda issue de milieu populaire et le jeune homme de bonne famille dont elle est amoureuse s'expriment dans le même registre de langue, ce qui enlève toute crédibilité à leur histoire, d'autant plus que le créole employé est un créole lontan ${ }^{22}$, qui ne tient pas compte des évolutions de la langue. Il sonne faux et ne suscite pas l'adhésion, bien au contraire. Un tel traitement de la langue, parce qu'il atteint l'identité même des téléspectateurs, les touche profondément et suscite des réactions émotionnelles fortes, qui sont autant de réponses au sentiment de stigmatisation ressenti. Il provoque les rires des plus jeunes, qui multiplient les parodies, et suscite un sentiment de malaise auprès des plus âgés. Entendre l'héroïne s'exprimer de la sorte entraîne un arrêt brutal du processus identificatoire et le rejet de la série : "C'est une honte de l'entendre parler comme ça ! » déplore une de nos interlocutrices ${ }^{23}$.

La station apprend ainsi à ses dépens que l'on ne touche pas impunément à la langue créole, dans un territoire qui reste marqué par la diglossie, même si le mélange des langues et l'hybridation constituent une forme émergente du parler à La Réunion. Le créole est toujours considéré comme la langue du cœur, de la vie quotidienne, du privé ; ce n'est pas encore la langue de l'espace public et il n'est toléré à la télévision que dans certaines occasions ${ }^{24}$. Une conclusion paradoxale s'impose : Thalia est une créole à qui l'on peut s'identifier à condition qu'elle ne parle pas créole !

\section{b) L'adaptation de Código Postal et sa réception}

Le second pari est à mettre au crédit de la direction nationale du Réseau France Outre-mer et de la Chaîne France Ô qui passent du statut de simple diffuseur de telenovelas à celui de co-producteurs ${ }^{25}$. La Baie des Flamboyants est ainsi une libre adaptation de Código Postal, une telenovela mexicaine de Televisa qui raconte les aventures sentimentales d'un groupe de jeunes sur les plages mexicaines.

\footnotetext{
${ }^{21}$ On évoque le cas de telenovelas dont le doublage en français, confié par souci d'économie à des étudiants québécois, était quasi incompréhensible.

${ }^{22}$ Un créole d'autrefois, figé dans le passé.

${ }^{23}$ Femme au foyer, 53 ans (voir note 11 ).

${ }^{24}$ Voir Bernard Idelson, "Le créole dans les médias réunionnais ", Hermès, n 40, 2004, p. $128-134$.

${ }_{25}$ Avec Jean-Luc Azoulay, producteur dans les années 1990 des séries d'AB Productions comme Hélène et les garçons.
} 
Tournée sous la direction de Gérard Espinasse ${ }^{26}$, la série domienne va proposer, en 100 épisodes de 26 minutes chacun, une nouvelle problématique, à savoir « le destin croisé de six familles et d'une jeunesse romantique née sous le soleil » ainsi que le résume la première ligne du synopsis. De nouveaux territoires sont donnés à voir, on quitte les plages mexicaines pour celles de la Guadeloupe, et de nouveaux comédiens sont engagés : ils sont originaires des DOM ou issus de la série adolescente Hélène et les garçons (TF1, 1992-94). L'objectif est de faciliter l'identification du public d'Outre-mer et de rajeunir ce dernier. Pour les promoteurs du projet, tous les ingrédients sont ainsi réunis pour que les audiences soient au rendez-vous.

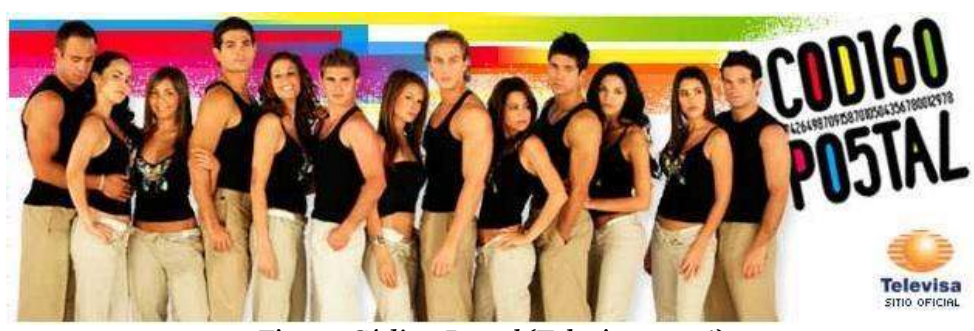

Fig. 7 : Código Postal (Televisa, 2006)

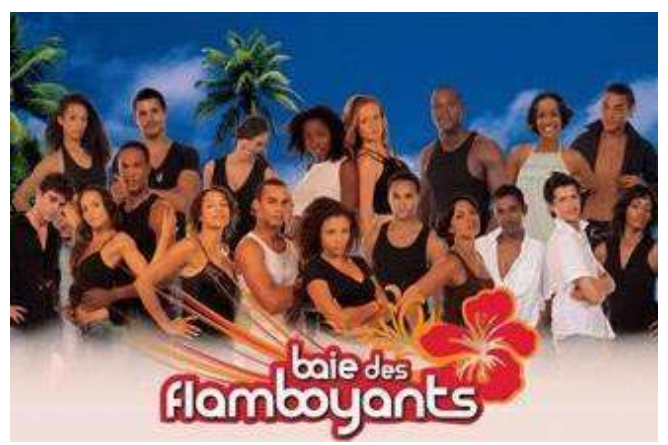

Fig. 8 : La Baie des Flamboyants (JLA-RFO, 2007)

L'étude de la réception de la première saison de La Baie des Flamboyants est envisagée ici en référence à un processus décliné en trois temps. Dans un premier temps, avant la rencontre avec le texte médiatique, on assiste à la mise en place du cadre de participation et d'interprétation ${ }^{27}$ défini par la chaîne. La « promesse » que RFO fait à

\footnotetext{
${ }^{26}$ Réalisateur des Vacances de l'amour (TF1, 1996-2004), une suite d'Hélène et les garçons. 27 Voir Jean-Pierre Esquenazi, «Les médias et leurs publics. Le processus de l'interprétation" in Stéphane Olivesi (dir.), Sciences de l’information et de la
} 
ses téléspectateurs est annoncée sur le site officiel de la station : $L a$ Baie des Flamboyants sera « la première fiction noire produite en France avec des comédiens majoritairement d'Outre-mer ${ }^{28} »$. Cette promesse est maintes fois rappelée par Luc Laventure, le directeur des antennes, qui précise le 30 septembre 2007, en direct sur le plateau du Journal Télévisé de RFO Guadeloupe : "C'est la première fois qu'il y aura une série noire française, une vraie série avec des Noirs, des Blacks, des Nègres, c'est important. » Notons que cette promesse est ensuite reprise par la presse quotidienne régionale antillaise alors que la presse nationale parle d'une production " symbole de la diversité », expression qui permet d'éviter le débat sur le racisme et la discrimination. À La Réunion, cette promesse n'a jamais été évoquée, peut-être parce qu'elle ne fait pas sens dans le contexte réunionnais ; les journalistes locaux ont focalisé l'attention sur la performance d'Émilie 29 , seule Réunionnaise retenue dans le casting de la Baie des Flamboyants après sa participation à la Star Academy.

Le moment de la confrontation avec le texte médiatique est le plus difficile à saisir pour les sociologues, car il est très intrusif et la présence des chercheurs crée "des manières de regarder différentes adaptées à ce que l'on perçoit comme étant la bonne manière d'être téléspectateur ", ce qui fausse les données ${ }^{30}$. Les mesures d'audience donnent une traduction statistique de cette rencontre avec le programme, rencontre très importante pour les producteurs. Les seuls chiffres livrés à la presse signalent que les premiers épisodes ont obtenu un accueil très positif du public guadeloupéen (61\% de taux d'audience), moins affirmé dans l'île voisine de la Martinique (40\%) et encore moins important à La Réunion (31\%). Si l'on se réfère aux seuls chiffres rendus publics, on note que plus on s'éloigne du lieu de tournage et plus l'intérêt du téléspectateur pour la série baisse. Mais les mesures d'audience ne disent rien du public lui-même, de ses émotions, du degré de son intérêt ou encore du contexte de réception ${ }^{31}$.

Nous partageons avec Dominique Pasquier la conviction qu' " on n'est pas un public, on se manifeste comme tel, pour un temps, face à d'autres, et selon des procédures adaptées à l'exigence des

communication. Objets, savoirs, discipline, Grenoble, Presses universitaires de Grenoble, 2006, p. 11-26.

${ }^{28}$ Édito de Luc Laventure, site RFO (page consultée le 5 juin 2008) :

http://baie-des-flamboyants.rfo.fr/indexfr.php?page $=$ news\&id_article $=81$

${ }_{29}$ Ainsi le Journal de l'île de la Réunion titre "Émilie vedette d’une fiction " (27 juillet 2007), «Premier clap de tournage avec Émilie " (7 août 2007), «Émilie Minatchy flamboyante » (24 novembre 2007).

$3^{30}$ Dominique Pasquier, «La télévision comme expérience collective : retour sur les Mondes de l'Art » in Alain Blanc et Alain Pessin (dir.), L'Art du terrain. Mélanges offerts à Howard Becker, Paris, L'Harmattan, 2004, p. 203 [p. 193-219].

${ }_{31}$ Voir Sabine Chalvon-Demersay, "La mesure du public: approche généalogique de l'audience télévisuelle », Quaderni, ${ }^{\circ}{ }^{3} 35,1998$, p. 45-51. 
situations $3^{2} »$. Nous sommes donc allés chercher ce public non pas auprès de ceux qui regardent l'émission et constituent l'audience, mais auprès de ceux qui revendiquent leur position spectatorielle. C'est le troisième temps de la réception, celui de l'après-visionnage, qui a ainsi particulièrement retenu notre attention, temps durant lequel le public a fait entendre sa voix via le forum du site officiel ${ }^{33}$ de la station ${ }^{34}$.

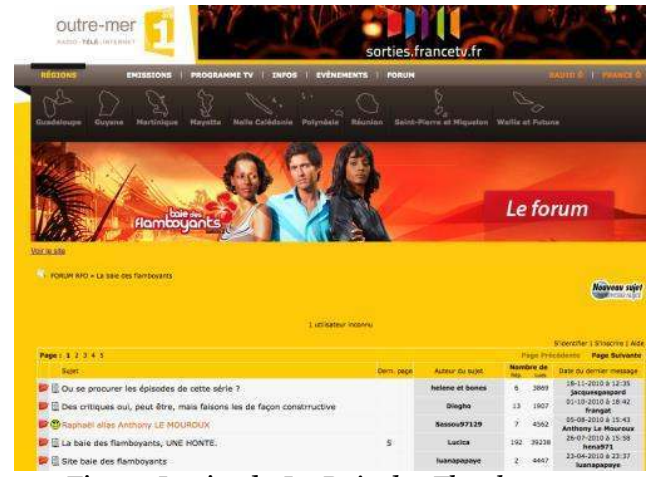

Fig. 9 : Le site de La Baie des Flamboyants

\section{L'émergence d'une communauté de réception}

Un constat s'impose à l'issue de nos premières investigations : le débat est presque exclusivement antillais, massivement guadeloupéen. De nombreux indices de légitimation de la parole sont communiqués par les internautes 35 . Certains mettent en avant leur lien avec l'une ou l'autre île des Antilles françaises en faisant référence à leur lieu de naissance, à l'ancrage généalogique et/ou racial de leur parentèle :

- Je suis issu d'un père blanc (Saintois) et d'une mère noire. Donc je suis métisse et je suis pour mon peuple (Black971)

- Je m'appelle laura et j'ai 15ans moi jss noir de mère et de père

d'origine indienne coté maternelle (Laura - 97122)

- Moi je suis une créole typique (père métisse indien, mère mélangée mulâtre et noir) (Floranella)

$3^{2}$ Pasquier, p. 202.

33 http://forums.la1ere.fr/la1ere/la-baie-des-flamboyants/liste_sujet-1.htm (consulté le $1^{\text {er }}$ juin 2011).

34 « Le site Baie des Flamboyants (BDF) a enregistré les meilleures audiences de tous les sites RFO, le forum BDF également a été le plus actif de tous les forums RFO. Par ailleurs des fans ont créé un autre site BDF et la page Facebook BDF est encore animée après 3 saisons » (Barbara Laup, Chef de projet internet, France télévision, entretien, mai 2011).

${ }_{35}$ Les interventions ont été retranscrites telles qu'elles ont été postées sur le site. 
- J'ai 27 ans, je suis antillaise (à moitié Guadeloupéenne, à moitié Martiniquaise) et je sais que les jeunes filles de cette série ne me ressemblent pas (Lucica)

Le lieu de résidence et la trajectoire personnelle insulaire sont particulièrement mis en évidence :

- Lorsque je circule en Guadeloupe en voiture dans ma belle

Guadeloupe, je ne vois que des noirs sur les trottoirs et une minorité de métisses (Black971)

- Cela fait 5 ans que je n'ai pas été en Guadeloupe et quand j'ai vu qu'il y avait une série faite en Guadeloupe, cela m'a fait très plaisir (Jolalaly)

D'autres indices d'appartenance et de connivence sont également donnés à voir, comme le choix d'un pseudonyme dans lequel figure le code postal et la référence à la Guadeloupe (Black971, Gwad971, siana97one) ou le recours au créole, le plus souvent réduit à un mot ou à un dicton au détour d'une phrase ${ }^{36}$ : « ke an mwen conten [...] lass kritiké lé on bon bitin ka rivé zot. »

Un premier survol des sujets proposés permet de distinguer deux types de discussions sur ce forum. Les téléspectateurs débattent de la série et de l'histoire qu'elle raconte. Une lecture critique et ludique prend place : elle consiste à traiter le programme comme une construction fictionnelle qui obéit aux règles du genre avec ses formules, ses conventions et ses schémas narratifs. On discute du déroulement de l'intrigue, du caractère ou du physique de tel ou tel personnage, du jeu des acteurs, on parie sur le déroulement de l'histoire. Ce type de lecture ${ }^{37}$ n'est pas exclusif d'une lecture référentielle ou réelle par laquelle les téléspectateurs connectent le programme avec leur vie quotidienne, mettent en relation les héros ou les intrigues avec des personnages ou des événements qui leur sont familiers et entrent dans le jeu de la fiction en imaginant leurs propres réactions s'ils étaient confrontés aux mêmes événements.

Les sujets soumis à la discussion sont nombreux et variés. Le débat sur les premiers épisodes témoigne de la curiosité des spectateurs au début de la diffusion. On échange également des avis sur l'acteur le plus beau, sur le rôle tenu par la méchante Johanna ; on fait des hypothèses sur la résolution de l'intrigue alors que la première saison touche à sa fin.

${ }^{36}$ Il s'agit ici de créole guadeloupéen que l'on pourrait traduire par « mon cœur est content [...] arrêtez de critiquer quand quelque chose de bien vous arrive ».

37 Les deux types de lecture évoqués ont été mis en évidence par Liebes et Katz (op. cit.) à propos du décodage de la série Dallas. 


\begin{tabular}{|l|c|c|}
\hline $\begin{array}{l}\text { Sujet } \\
\text { (situation au 15 septembre 2008) }\end{array}$ & $\begin{array}{c}\text { Nombre } \\
\text { réponses }\end{array}$ & $\begin{array}{c}\text { Nombre } \\
\text { lectures }\end{array}$ \\
\hline $\begin{array}{l}\text { Presque la fin... comment la voyez vous ? et } \\
\text { après? }\end{array}$ & 34 & 1205 \\
\hline Le plus beau? & 32 & 1565 \\
\hline Spécial Johanna & 62 & 4483 \\
\hline Les premiers épisodes & 60 & 4089 \\
\hline Allez parlons du côté positif un peu & 106 & 4303 \\
\hline $\begin{array}{l}\text { La baie des flamboyants, une vraie } \\
\text { HONTE pour notre peuple }\end{array}$ & $\mathbf{8 8}$ & $\mathbf{5 1 0 1}$ \\
\hline La baie des flamboyants unE HONTE & $\mathbf{3 8}$ & $\mathbf{1 0 ~ 9 1 9}$ \\
\hline
\end{tabular}

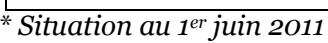

Deux sujets aux intitulés très proches se distinguent par le nombre important de réponses qu'ils suscitent et plus encore par celui des lectures qui en sont faites. Il s'agit de « La baie des flamboyants, UNE HONTE » et « La baie des flamboyants, une vraie HONTE pour notre peuple ». Ces sujets se singularisent également par la virulence, la violence et la passion qui animent les interactions $3^{38}$ : ici la polémique a remplacé le débat. Les téléspectateurs réagissent essentiellement en référence à la promesse du feuilleton dont les deux thématiques majeures étaient la promotion de l'Outre-mer et la référence à la diversité focalisée sur la couleur des acteurs (« une série avec des Noirs »). Et manifestement le résultat n'est pas à la hauteur des attentes et des espoirs d'une partie du public qui est fort mécontent et le fait savoir : " après le tapage médiatique qu'on nous a servi on est en droit d'exiger quelque chose qui nous ressemble39 ».

Les interventions expriment un fort sentiment de déception à la réception de ce produit culturel, lu et investi par certains comme un documentaire plus que comme une fiction. Ainsi, le débat polémique qui s'engage sur le forum est particulièrement focalisé sur la couleur des acteurs, jugés trop métissés pour être véritablement représentatifs de la Guadeloupe : «Lorsque je circule en Guadeloupe, je ne vois que

38 « Le forum a suscité de vives réactions (notamment des propos à caractère raciste qui ont été effacés) dès la diffusion des premiers épisodes. On a rapidement changé le mode de modération du forum et dans un premier temps aucun commentaire n'apparaissait sans être validé en amont. On était parti sur un modèle d'interactivité comme Plus belle la vie, loin d'imaginer des retours aussi agressifs et des débats houleux qui nous empêcheraient de sortir du modèle de base du forum » (Barbara Laup, Chef de projet internet, France télévision, entretien, mai 2011).

39 Posté sur le forum http://forums.la1ere.fr/la1ere/La-baie-des-flamboyants/flamboyantsune-honte-sujet_13_2.htm ainsi que les citations suivantes. 
des noirs sur les trottoirs et une minorité de métisses. Voilà la réalité et non votre photo de famille d'acteurs venus d'ailleurs où le métissage et la dépigmentation de la peau est trop prononcée », remarque un internaute. Il estime que " cette série est une insulte pour le peuple guadeloupéen » et son intervention est représentative des critiques émises. S'ouvre un débat passionné entre ceux qui défendent la série perçue comme un objet fictionnel (certes perfectible en tant que produit) et ceux qui la critiquent principalement parce qu'elle donne une image tronquée de la Guadeloupe et de ses habitants.

Le débat sur la couleur des acteurs et leur représentativité, et les controverses autour du degré de métissage de la population guadeloupéenne et de sa typologie, font particulièrement sens dans une île où « la ligne de couleur 40 » constitue un principe de distinction et de hiérarchie sociale encore très actif. Les enjeux de définition sur ce qui est ou non représentatif de la culture, de la population, des paysages et des rapports sociaux "guadeloupéens », s'avèrent particulièrement importants pour ce public qui s'est senti fortement interpellé par les promesses du feuilleton. Une communauté de réception pour laquelle ce débat fait sens est ainsi apparue sur ce site, transformé pour un temps en une arène où s'affrontent avec ardeur, voire violence, des définitions plurielles à propos d'un «commun » territorialement et historiquement situé : les Antilles en général, et la Guadeloupe en particulier. Plus que la raison, c'est la passion qui domine les échanges dans cet espace public ${ }^{41}$ dont les dimensions phénoménale, émotionnelle et sensible sont dominantes ; une émotion que ne partage pas le public réunionnais, inscrit dans une socio-histoire différente et qui ne se retrouve pas dans cette problématique identitaire de la ligne de couleurs.

Pour conclure provisoirement, il nous faut interroger la perspective domino-centrée des sociologues critiques. En marge des grandes industries culturelles productrices de séries, un espace de jeu existe au niveau de la production nationale française d'Outre-mer ${ }^{2}$ qui, on l'a vu, a fait le pari de la traduction, de la relocalisation et de

$4^{4}$ Jean-Luc Bonniol, La Couleur comme maléfice. Une illustration créole de la généalogie des « Blancs » et des « Noirs », Paris, Albin Michel, 1992.

${ }^{41} \mathrm{Au}$ sens que lui donne Hannah Arendt, Condition de l'homme moderne, Paris, Plon, 1994. $4^{2}$ C'est le cas pour la Saison 1 de La Baie des Flamboyants. Les Saisons 2 et 3 ont été coproduites par France Télévision, JLA et Televisa, dont l'expertise a permis d'améliorer le produit et de le vendre au public francophone mais également en Chine, en Afrique et en Grèce. Puis les droits ont été rachetés par la toute-puissante entreprise brésilienne TV Globo, qui a fait traduire les dialogues en portugais pour revendre la série intitulée Praira de galera au marché lusophone. Enfin, un spin-off intitulé "Les Flamboyants » a été tourné en 2011 dans un hôtel à Saint Martin avec de jeunes acteurs, « ceux qui faisaient le cœur de l'histoire de Baie des Flamboyants », a déclaré Jean-Luc Azoulay au moment du lancement de la série sur le réseau Antilles-Guyane Première (France Antilles, le 9 septembre 2011) (http://www.guadeloupe.franceantilles.fr/loisirs/une-tv-mag/en-vue-lesflamboyants-saison-1-vent-de-fraicheur-09-09-2011-137973.php) 
l'adaptation aux spécificités culturelles d'un public imaginé. Leurs propositions ne rencontrent pas toujours le succès escompté; un certain nombre de leurs paris échouent ou échappent à l'intentionnalité de leurs producteurs et ne trouvent pas toujours leurs publics. Pour mieux saisir ce public, il nous faut complexifier la question de la réception et dépasser la dyade texte-lecteur, qui a trop longtemps négligé les dimensions sociales et temporelles d'un processus ternaire. Si l'étude de « l'avant » n'est pas à négliger, car s'y effectue un important travail de cadrage de l'interprétation, « l'après » retient toute notre attention. C'est à ce moment-là qu'un public se donne à voir et, dans le même temps, donne à voir les conflits de définitions identitaires qui sont au cœur du débat engagé dans la sphère publique constituée ici par le forum de discussion. Étudier le public qui se mobilise et émerge à cette occasion permet d'éviter un important biais des enquêtes de réception : interroger des individus que les sociologues ventriloques constituent en public «a priori ». Comme le souligne Daniel Dayan, "en donnant la parole à des spectateurs qui ne l'ont pas sollicitée, en posant la légitimité de cette parole, on amène ceux-ci à rendre explicites [...] des réactions qui, souvent, ne sont ni discursives, ni argumentées ", ce qui revient "à inventer une fiction de public et à lui donner la parole »43. Il nous faut donc observer ces publics au moment où ils apparaissent dans l'espace public, et Internet nous offre un territoire d'observation privilégié et encore trop peu exploré. S'y révèlent des communautés de réception très différenciées pour lesquelles le débat fait sens. La signification des œuvres intervient dans cette opération de co-construction du sens entre une proposition médiatique et des téléspectateurs qui s'en saisissent et en débattent pour construire leurs identités personnelles ou collectives ${ }^{44}$, qu'elles soient sexuée, générationnelle, de classe, mais également, comme on a pu le voir ici, qu'elles soient linguistique, culturelle, ethnique, ultramarine, locale, nationale ou globale. La démarche nous a, finalement, permis d'inscrire les pratiques de réception dans des contextes socialement et culturellement situés et de faire éclater la catégorie «Outre-mer» dans laquelle les publics ultramarins sont encore trop rapidement enfermés.

\section{Bibliographie}

ARENDT Hannah, Condition de l'homme moderne, Paris, Plon, 1994.

\footnotetext{
43 Daniel Dayan, «Télévision, le presque-public », Réseaux nº10o, 200o, p. 438 [p. 427456].

${ }_{44}$ Serge Proulx et Éliane Wolff, « La réception sociale de la télévision », in Michel Watin, (dir), Espace public et communication, Univers créoles 1, Paris, Economica-Anthropos, 2001, p. 129-156.
} 
BIANCHI Jean, « La promesse du feuilleton. Structure d'une réception télévisuelle », Réseaux, ${ }^{\circ} 39,1990$, p. 9-18.

Bonniol Jean-Luc, La Couleur comme maléfice. Une illustration créole de la généalogie des «Blancs» et des «Noirs », Paris, Albin Michel, 1992.

BouQuillon Philippe, «La réception des telenovelas brésiliennes en France », Intercom, vol. XV, ${ }^{\circ}{ }^{1}$, 1992, p. 98-117.

Chalvon-Demersay Sabine, «La mesure du public: approche généalogique de l'audience télévisuelle », Quaderni, nº35, 1998, p. 4551 .

CSA, Bilan de la société nationale de programme Réseau France outre-mer, 2002 (lien consulté le 30 septembre 2011), www.csa.fr/content/download/17291/317471/file/bilRFO.pdf

DAYAN Daniel, «Les mystères de la réception. Ethnologues, missionnaires, ventriloques », Le Débat, n 71, 1992/4, p. 146-162.

DAYAN Daniel, « Télévision, le presque-public », Réseaux, n¹00, 2000, p. 427-453.

EsQUENAZI Jean-Pierre, « Les médias et leurs publics. Le processus de l'interprétation » in Stéphane Olivesi (dir.), Sciences de l'information et de la communication. Objets, savoirs, discipline, Grenoble, Presses universitaires de Grenoble, 2006, p. 11-26.

HOAREAU Stéphanie, «À la recherche des publics de Marimar à la Réunion ", mémoire de maîtrise en SIC, sous la direction d'Éliane Wolff, Université de la Réunion, 2005.

IDELSON Bernard, « Le créole dans les médias réunionnais », Hermès, $\mathrm{n}^{\circ} 40,2004$, p. 128-134.

LiEbes Tamar et Elihu Katz, The Export of Meaning: Cross-Cultural Readings of 'Dallas', New York, Oxford University Press, 1990.

MAcÉ Eric, "Qu'est-ce qu'une sociologie de la télévision ? (2) Esquisse d'une théorie des rapports sociaux médiatisés. Les trois moments de la configuration médiatique de la réalité : production, usages, représentations.», Réseaux, ${ }^{\circ}{ }^{\circ} 105,2001$, p. 199-242.

PASQUIER Dominique, «La télévision comme expérience collective : retour sur les Mondes de l'Art » in Alain Blanc et Alain Pessin (dir.), 
L'Art du terrain. Mélanges offerts à Howard Becker, Paris, L'Harmattan, 2004, p. 193-219.

ProulX Serge et Éliane WolfF, «La réception sociale de la télévision », in Michel Watin (dir.), Espace public et communication, Univers créoles 1, Paris, Economica-Anthropos, 2001, p. 129-156.

Quotidien de La Réunion, «Telenovelas. Pourquoi La Réunion en est dingue », dossier, 2 novembre 2010, Saint Denis de la Réunion, 2010.

Thomas Erika, Les Telenovelas entre fiction et réalité, Paris, L'Harmattan, 2003.

Thomas Erika, "Les telenovelas: une passion brésilienne », Ina Global, La Revue des industries créatives et des médias (consulté le 20 juin 2011) http://www.inaglobal.fr/television/article/les-telenovelasune-passion-bresilienne?tq=12\#intertitre-2

WolfF Éliane, «De Marimar à Rosalinda péi (pays), l'expérience réunionnaise », in Eric Maigret et Guillaume Soulez (dir.), Les Raisons d'aimer les séries télé, MédiaMorphoses, numéro spécial, janvier 2007, p. 59-63.

WolfF Éliane, «Publics, réception médiatique et contexte d'usage : retour sur 10 années de recherches " in Bernard Idelson (dir.), Journalismes dans l'Océan Indien: espaces publics en questions, Paris, L'Harmattan, 2008, p. 53-67.

WoLFF Éliane, « Familles en mutations, famille en questions » in Michel Watin (dir.), La Réunion, une société en mutation, Univers créoles 7, Paris, Economica-Anthropos, 2010, p.81-102. 